\title{
An Essay on Self-explanatory Computational Intelligence: A Linguistic Model of Data Processing Systems
}

\author{
Jose M. Alonso \\ Centro de Investigación en \\ Tecnoloxías da Información (CiTIUS) \\ University of Santiago de Compostela, \\ Santiago de Compostela, Spain \\ josemaria.alonso.moraleusc.es
}

\author{
Gracian Trivino \\ Phedes Lab, \\ Asturias, Spain \\ gracian.trivino@phedes.com
}

\begin{abstract}
Computational processes are increasingly more powerful and complex but also more difficult to understand by humans. Considering that Natural Language is a suitable tool for describing human perceptions, building selfexplanatory computational systems ready to communicate with humans in Natural Language becomes a hot challenge. Based on ideas taken from Cognitive Science, we propose a novel model to facilitate achieving this goal. We consider the computer as a metaphor of the mind and we use references from Philosophy, Neurology, Linguistics, Anthropology and Sociology to provide a structure of different components that allow coping with the complexity of generating linguistic descriptions about computational processes. We illustrate the use of this model with several examples.
\end{abstract}

\section{Introduction}

Currently, computational systems allow accessing huge amounts of data about the phenomena in their environment. Nevertheless, users and engineers demand tools to reduce the size and complexity of these data into more friendly tractable dimensions. We, human beings, use Natural Language for describing our perceptions and also for construing our experience (Halliday and Matthiessen, 1999).

Indeed, nowadays, computers can use Natural Language to generate linguistic descriptions of the complex phenomena in their environment. The new challenge (Gunning, 2016) is to build selfexplanatory computational systems, i.e., computa- tional systems able to describe linguistically their own functioning.

There are several related research lines dealing with Argument Technology (Walton et al., 2008), Natural Language Generation (Reiter and Dale, 2000) and more specifically with Linguistic Description of Data (Ramos-Soto et al., 2016). Moreover, we have already proved the benefits of using Natural Language for building explainable fuzzy systems (Alonso et al., 2017).

When we deal with describing computational processes, we need a way of representing the meaning of the related linguistic descriptions, i.e., a way of organizing and coping with their complexity that would make them easier to understand.

The work presented in this paper contributes to a long term research project, the so-called Linguistic Description of Complex Phenomena (Trivino and Sugeno, 2013). The main contribution of this paper is a systemic model of the process performed by human beings and computers as Data Processing Systems (DPS) for producing new information from input data. We propose to use this model to organize the meaning of linguistic descriptions about the different components of computational processes.

This is a general model including few processes associated with specific families of linguistic expressions. The model deals with a classification of the main DPS activities and the more adequate linguistic expressions to describe them. Here, we present only a brief description of the model components but hopefully enough to provide the reader with an insight about the possibilities of the idea.

In Section 2 we present some preliminary con- 
cepts which are required to understand the proposal drawn in Section 3. Section 4 provides some illustrative examples. Finally, conclusions and future work are sketched in Section 5.

\section{Several Concepts from Cognitive Science}

One of the main premises of Cognitive Science asserts that the computer can be considered a metaphor of the mind (Gardner, 1987). This metaphor can be used in both directions, the computer to create an idea of how the mind works but also we can use our knowledge about the mind to organize linguistic descriptions about how computers work. With this last regard we recall several ideas from disciplines belonging to Cognitive Science.

From Philosophy, according with Popper, the universe where humans beings live can be divided into three worlds (Popper and Eccles, 1977):

- The world of the physical objects (W1).

- The world of the perceived objects (W2).

- The world of the mental objects (W3).

From Neurology, according with (Damasio, 2003), Natural Evolution has built the hierarchical control system of the human behavior by aggregating step by step a series of successive layers:

- The primitive layer, located in the inner part of the brain, is dedicated to immune responses, basic reflexes and metabolic regulation.

- Control related with pain and pleasure.

- Control based on drives and motivations.

- Control based on emotions and feelings.

- On the top of this hierarchy of control mechanisms we have the rationality. This is part of the most evolved behavior control mechanism that is based on using Natural Language.

From Linguistics, Systemic Functional Linguistics (Halliday and Matthiessen, 1999) provides a classification of the human activities into four main types and subtypes:

- Being: (1) Identifying, (2) Ascribing, and (3) Existing.
- Sensing: (1) Seeing, (2) Feeling, (3) Thinking, and (4) Wanting.

- Doing: (1) Doing to/with, (2) Happening, and (3) Behaving.

- Saying: there are no subtypes here.

\section{A Linguistic Model of Data Processing Systems}

This section presents the main contribution in this paper. We apply a systemic approach inspired by the ideas introduced in Section 2. We have defined a model which describes the basic components that both humans and computers see as Data Processing Systems (DPS). Fig. 1 shows a data flow diagram of the model. Rectangles correspond to data structures and ovals represent processes. In the rest of the section we describe the main components in the model. First, Section 3.1 describes the data structures. Second, Section 3.2 introduces the processes.

\subsection{Data Structures}

\subsubsection{External Phenomena (W1)}

This is the external world that forms the system environment. Using their sensors, DPS try to obtain useful data that are needed to perform their goals. Notice that most of the phenomena in W1 are beyond the limits of human/computers perception and understanding capacities.

We identify two main components in this world: (1) World of physical objects, and (2) World of cultural objects.

The World of physical objects corresponds with the first world described by (Popper and Eccles, 1977). Both human body and robot hardware are part of this world which is only accessed through physical introspection (proprioceptive sense).

Currently, as a result of the civilization process, the environment from which DPS perceive relevant phenomena and they must make their decisions is not only physical but also cultural. We call this World of cultural objects. According with Anthropology (Tomasello, 1999) and Sociology (Berger and Luckmann, 2011) this world is built by humankind following the Natural Evolution and using Natural Language. 


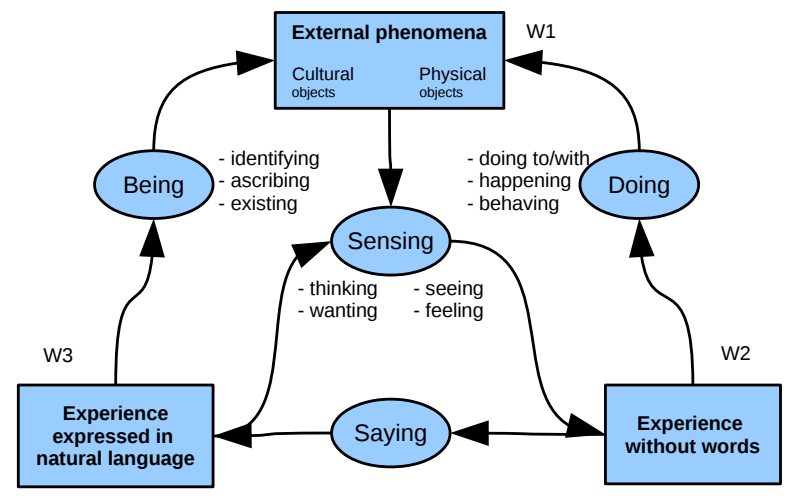

Figure 1: Data flow diagram of a linguistic model for Data Processing Systems (DPS).

\subsubsection{Experience Without Words (W2)}

It takes place in the internal world that is the domain of drives, emotions and feelings for humans. It is made up of internal images without words and corresponds with the Popper's second world, i.e., the world of the perceived objects. In computational systems, these objects are related to raw data, i.e., data that computers capture for driving reactive behaviors but that still need to be processed before becoming useful information. For example a video stream or a temporal series of temperatures coming out from a chemical process.

\subsubsection{Experience Expressed in Natural Language (W3)}

This data structure corresponds with the Popper's third world. In accordance with Neurology, it is part of the human consciousness (Damasio, 2010). The related information is produced in humans by using the rationality that is supported by the newest part of the human brain, the neocortex. This is part of the most evolved behavior control mechanism that is based on using the Natural Language. On the other hand, in computers, it is produced by using the highest levels of their computational architecture (Trivino et al., 2009).

\subsection{Processes}

It is noteworthy that the names of these processes are labels representing a classification (hierarchical structure) of possible linguistic expressions. During their application in the model, each specific situation will be described by using specific linguistic expressions belonging to these categories.

\subsubsection{Sensing}

This process allows DPS to obtain data about the External phenomena. Seeing is a specialized function or sensor (as sight, hearing, smell, taste, or touch in humans) by which DPS sense (obtain or receive) external or internal stimuli. It allows creating images of objects in the surrounding environment. With Feeling the system translates the information coming from external phenomena into emotions and feelings that will influence/condition the related behavior. In computers, it is used for reactive control. In Fig. 1 they correspond with the process $W 1$ $\rightarrow W 2$. Thinking and Wanting corresponds with the feedback $W 3 \rightarrow W 2$. DPS can distinguish between positively and negatively evaluated information and modify accordingly the related behavior. With Saying they close an internal control loop.

\subsubsection{Doing}

In Fig. 1, it corresponds with the process $W 2 \rightarrow$ W1. It includes the processes of physical acting and can be described by linguistic expressions like Doing to/with, Happening, and Behaving.

\subsubsection{Saying}

It is the process of generating linguistic descriptions of images or raw data. In Fig. 1, it corresponds with the process $W 2 \rightarrow W 3$. This is related to the research on Linguistic Descriptions of Complex Phenomena, e.g., (Conde-Clemente et al., 2017).

\subsubsection{Being}

In Fig. 1, it corresponds with the process $W 3 \rightarrow$ W1. It can be described by linguistic expressions like Identifying, Ascribing, and Existing. Using this 
process DPS create new objects and modify the objects in W1. Note that this is the mechanism used by DPS to send messages to other DPS.

\section{Examples}

In this section, with the aim of illustrating how to apply the model presented in Section 3, we describe briefly the sequences of processes followed in two examples of human behavior and in one example of computational processing.

\subsection{Reactive Behavior}

Let's suppose we observe a reactive behavior in a woman. She shows a typical reactive activity when the light of the sun is disturbing her to see something:

- She feels the sun on her eyes (sensing-seeingfeeling).

- She moves her head looking for the sun position (doing-behaving).

- She uses her hand to shadow her eyes (doingdoing with).

\subsection{Deliberative Behavior}

Here, the observed subject (a musician) shows a more sophisticated set of activities that consists of composing music:

- He listens to a bird through the window (sensing-seeing).

- He feels certain emotion (sensing-feeling).

- He plays the piano (doing-doing with).

- He expresses this music using the musical notation (saying).

- He publishes a piano score and makes the music available to others by creating a new object in their external world (being-existing).

\subsection{Computational System Behavior}

A computational system (DPS) monitors the movements of clients into a supermarket:

- It detects a change in the shopping entrance (Sensing).
- It changes the internal state to "detected new client" and stores the related image [experience without words].

- It moves the camera to follow the client (doing).

- It builds a map with different positions and timestamps (Saying).

- It detects the client is going out (Sensing).

- It sends a linguistic report to the store manager informing about business details (Being).

\section{Conclusions}

The presented model is the result of a multidisciplinary research and it is part of a long term project in the research line of Linguistic Descriptions of Complex Phenomena.

In general, computational processing of data are complex phenomena. The idea is that computers use a metaphor to describe their internal processes. The human user is helped to understand the computer processes by using the same terminology that the user uses to describe his/her own activities.

We have focused this paper on developing a model for meaning representation rather than in how to express this meaning with linguistic expressions that should be customized for each specific user. The description presented in this paper provides just a general insight about the main components of the model and how they are interrelated. The next step includes to analyze and to describe in depth the three data structures and the four processes.

This paper can be useful to researchers by providing them with a first idea about how to organize the meaning representation of linguistic descriptions of computational processes of data.

\section{Acknowledgments}

This work has been funded by TIN2014-56633-C31-R and TIN2014-56633-C3-3-R projects from the Spanish "Ministerio de Economía y Competitividad". Financial support from the Xunta de Galicia (Centro singular de investigación de Galicia accreditation 2016-2019) and the European Union (European Regional Development Fund - ERDF), is gratefully acknowledged. 


\section{References}

J. M. Alonso, A. Ramos-Soto, E. , and K. van Deemter. 2017. An exploratory study on the benefits of using natural language for explaining fuzzy rule-based systems. In IEEE International Conference on Fuzzy Systems (FUZZ-IEEE), pages 1-6, Naples, Italy.

P. L. Berger and T. Luckmann. 2011. The Social Construction of Reality: A Treatise in the Sociology of Knowledge. Open Road Media.

P. Conde-Clemente, Jose M. Alonso, E.O. Nunes, A. Sanchez, and G. Trivino. 2017. New types of computational perceptions: Linguistic descriptions in deforestation analysis. Expert Systems With Applications, 85:46-60.

A. R. Damasio. 2003. Looking for Spinoza: Joy, Sorrow, and the Feeling Brain. Harvest books. A Harvest Book.

A. R. Damasio. 2010. Self comes to mind. Constructing the conscious brain. Pantheon books.

H. Gardner. 1987. The Mind's New Science: A History of the Cognitive Revolution. Psychology: History. BasicBooks.

D. Gunning. 2016. Explainable Artificial Intelligence (XAI). Technical report, Defense Advanced Research Projects Agency (DARPA), Arlington, USA. DARPABAA-16-53.

M. A. K. Halliday and M. I. M. Matthiessen. 1999. Construing Experience through Meaning: A Languagebased Approach to Cognition. Continuum; Study ed edition (June 3, 2006).

K. R. Popper and J. C. Eccles. 1977. The Self and Its Brain. Springer-Verlag, Berlin.

A. Ramos-Soto, A. Bugarín, and S. Barro. 2016. On the role of linguistic descriptions of data in the building of natural language generation systems. Fuzzy Sets and Systems, 285:31-51.

E. Reiter and R. Dale. 2000. Building natural language generation systems, volume 33. MIT Press.

M. Tomasello. 1999. The cultural origins of human cognition. Harvard, New York.

G. Trivino and M. Sugeno. 2013. Towards linguistic descriptions of phenomena. International Journal of Approximate Reasoning, 54(1):22-34.

G. Trivino, L. Mengual, and A. Van der Heide. 2009. Towards an architecture for semiautonomous robot telecontrol systems. Information Sciences, 179(23):39733984.

D. Walton, C. Reed, and F. Macagno. 2008. Argumentation Schemes. Cambridge University Press. 